\title{
PONTRJAGIN NUMBERS OF MAPS
}

\author{
BY P. E. CONNER ${ }^{1}$ \\ Communicated December 6, 1962
}

We consider an integral valued $K$-genus; that is, a ring homomorphism $K: \Omega \rightarrow Z$ which carries the unit of the oriented Thom bordism ring into 1 . The integrality of certain genera, such as the $A$-genus or the $L$-genus [6, p. 13], provides some divisibility conditions which must be satisfied by the Pontrjagin numbers of closed manifolds. For the study of the oriented bordism module $\Omega_{*}(X)$ of a space the Pontrjagin numbers of a map of a closed oriented manifold into $X$ were defined in [4]. We shall explore in this note the corresponding divisibility conditions, caused by the integrality of $K$, which must be satisfied by the Pontrjagin numbers of such maps.

For each space $X$ let $Q(X)$ be the collection of all additive rational valued homomorphisms $\Gamma: \Omega_{*}(X) \rightarrow Q$ which are compatible with the $K$-genus in the sense that for $\left[M^{n}, f\right] \in \Omega_{n}(X), \quad\left[V^{n}\right] \in \Omega_{m}$, $\Gamma\left(\left[M^{n}, f\right]\left[V^{m}\right]\right)=\left(\Gamma\left(\left[M^{n}, f\right]\right)\right)\left(K\left(\left[V^{m}\right]\right)\right)$. The $Q(X)$ is a linear space over the rationals. Let $K_{j}\left(p_{1}, \cdots, p_{j}\right)$ be the multiplicative sequence of homogeneous polynomials with rational coefficients which determine $K\left[6\right.$, p. 80]. For each closed oriented manifold $M^{n}$, let $k\left(M^{n}\right)$ $\in H^{*}\left(M^{n} ; Q\right)$ be the cohomology class $\left(1, K_{1}\left(p_{1}\right), K_{2}\left(p_{1}, p_{2}\right), \cdots\right.$, $\left.K_{j}\left(p_{1}, \cdots, p_{j}\right), \cdots\right)$, where $p_{j} \in H^{4 i}\left(M^{n} ; Q\right)$ is a Pontrjagin class of the tangent bundle to $M^{n}$. We define a linear homomorphism $\Psi: H^{* *}(X ; Q) \rightarrow Q(X)$. For $c \in H^{* *}(X ; Q)$ we set $\Gamma_{c}\left(\left[M^{n}, f\right]\right)$ $=\left\langle k\left(M^{n}\right) f^{*}(c), \sigma_{n}\right\rangle$, where $\sigma_{n}$ is the orientation class and the brackets denote the cap product $\sigma_{n} \cap k\left(M^{n}\right) f^{*}(c)$ followed by the augmentation $\epsilon_{*}: H_{*}(X ; Q) \rightarrow Q$. A CW-complex $X$ is of finite type if and only if every skeleton $X^{(k)}$ is finite. We shall only consider such spaces.

1. If $X$ is a CW-complex of finite type then $\Psi(c)=\Gamma_{c}$ is a linear isomorphism between $H^{* *}(X ; Q)$ and $Q(X)$.

A $\Gamma \in Q(X)$ is integral valued if and only if $\Gamma\left(\Omega_{*}(X)\right) \subset Z$. This additive subgroup $z(X) \subset H^{* *}(X ; Q)$ consists of cohomology classes $c$ for which $\Psi(c)$ is integral valued. For each space the subgroup $z(X)$, which is not always homogeneous, represents the divisibility conditions imposed by $K$ on the Pontrjagin numbers of maps of closed oriented manifolds into $X$. Another interpretation is the consideration of $z(X)$ as the group of virtual $K$-genera on $X[6$, p. 85]. Under the homomorphism induced by $f: X \rightarrow Y, f^{*}(z(Y)) \subset z(X)$. If

1 This author is an Alfred P. Sloan Fellow and was partially supported by the U. S. Army Research Office. 
$f_{*}: \Omega_{*}(X) \rightarrow \Omega_{*}(Y)$ is surjective, then $\left(f^{*}\right)^{-1}(z(X))=z(Y)$. If $f_{*}: \Omega_{*}(X)$ $\rightarrow \Omega_{*}(Y)$ is injective, then $f^{*}(z(Y))=z(X)$. If $H_{*}(X ; Z)$ has no torsion, then $z(X)$ is a subring. For the $n$-sphere, $z\left(S^{n}\right)$ is the image of $H^{*}\left(S^{n} ; Z\right)$ $\rightarrow H^{*}\left(S^{n} ; Q\right)$ under the coefficient homomorphism, a fact noted by Milnor for the $L$-genus [7, Theorem 40], and which suggested this note. An application of $z(X)$ is based on the observation that a spherical cohomology class in $H^{* *}(X ; Z)$ also belongs to $z(X)$. From the structure of $z(X)$ it is possible to read off nontrivial conditions necessary that a cohomology class be spherical.

For a finite CW-complex the oriented cobordism groups $\Omega^{m}(X)$ were defined by Atiyah to be the cohomology groups of $X$ with coefficients in the Thom spectrum $M S O[1 ; 3]$. In addition, the pairing of $M S O$ with itself given by the natural maps $M S O(p) \wedge M S O(q)$ $\rightarrow M S O(p+q)$ induces on $\Omega^{*}(X)$ a graded anticommutative ring structure. Also there is a cap product pairing of $\Omega_{n}(X)$ with $\Omega^{m}(X)$ into $\Omega_{n-m}(X)$ which is formally analogous to the cap product between homology and cohomology [8]. With the aid of this pairing we may define a homomorphism $m: \Omega^{*}(X) \rightarrow z(X) \subset H^{*}(X ; Q)$. Let $\epsilon_{*}: \Omega_{*}(X)$ $\rightarrow \Omega$ be the augmentation induced by mapping $X$ onto a point. For $\alpha \in \Omega^{m}(X)$ we set $\Gamma_{\alpha}\left(\left[M^{n}, f\right]\right)=K\left(\epsilon_{*}\left(\left[M^{n}, f\right] \cap \alpha\right)\right) \in Z$. We define $m$ by $m(\alpha)=\Psi^{-1}\left(\Gamma_{\alpha}\right)$.

2. For every finite CW-complex, $m: \Omega^{*}(X) \rightarrow H^{*}(X ; Q)$ is a ring homomorphism. If $H_{*}(X ; Z)$ has no torsion, then $m\left(\Omega^{*}(X)\right)=z(X)$.

This $m$ commutes with homomorphisms induced by maps, but not with suspension. The Thom-Atiyah duality theorem [1] asserts the existence, for a closed oriented manifold, of a canonical isomorphism $\Omega_{n-m}\left(M^{n}\right) \simeq \Omega^{m}\left(M^{n}\right)$. In fact, for $\alpha \in \Omega^{m}\left(M^{n}\right)$ the dual bordism class is $\left[M^{n}, f\right] \cap \alpha$. In view of this duality we may impose on $\Omega_{*}\left(M^{n}\right)$ the structure of an intersection algebra over $\Omega$. In addition we have a ring homomorphism $\Omega_{*}\left(M^{n}\right) \rightarrow z\left(M^{n}\right)$ which we also denote by $m$. This homomorphism is related to the $\Omega$-module structure by the rule

$$
m\left(\left[M^{n}, f\right]\left[V^{m}\right]\right)=\left(m\left(\left[M^{n}, f\right]\right)\right)\left(K\left(\left[V^{m}\right]\right)\right) .
$$

For a map $f: M^{n+k} \rightarrow M^{n}$ between closed oriented manifolds, let $f_{*}: H^{i+k}\left(M^{n+k} ; Q\right) \rightarrow H^{i}\left(M^{n} ; Q\right)$ be the Gysin cohomology homomorphism induced by Poincare duality. We define a new $f_{\sharp}: H^{*}\left(M^{n+k} ; Q\right)$ $\rightarrow H^{*}\left(M^{n} ; Q\right)$ by the formula

$$
f_{\#}(c) k\left(M^{n}\right)=f_{*}\left(c k\left(M^{n+k}\right)\right) .
$$

3. For any map $f: M^{n+k} \rightarrow M^{n}$ between closed oriented manifolds, $f_{f}\left(z\left(M^{n+k}\right)\right) \subset z\left(M^{n}\right)$ and the diagram 


$$
\begin{aligned}
& f_{*}: \Omega_{*}\left(M^{n+k}\right) \rightarrow \Omega_{*}\left(M^{n}\right) \\
& f_{\#}: H^{*}\left(M^{n+k} ; Q\right) \rightarrow H^{*}\left(M^{n} ; Q\right)
\end{aligned}
$$

is commutative.

This last result is completely elementary, however it was suggested by the formalism in $[5]$. We note in particular that $m\left(\left[M^{n+k}, f\right]\right)$ $=f \#(1)$. At this point we note that an application of $z(X)$ to the study of spherical homology classes is based on the observation that for any map $f: S^{n} \rightarrow X$ and any $c \in z(X),\left\langle f^{*}(c), \sigma_{n}\right\rangle=\left\langle c, f_{*}\left(\sigma_{n}\right)\right\rangle$ is an integer.

Let $\beta(t)$ be the characteristic power series of the $K$-genus [6, p. 10]. Let $\alpha(t)=t / \beta\left(t^{2}\right)$, so for the $L$-genus, $\alpha(t)=\tanh (t)$, while for the $A$-genus, $\alpha(t)=(\sinh 2 t) / 2$. In the infinite complex projective space, $z(C P(\infty))$ is generated by 1 and $\alpha(x)$, where $x \in H^{2}(C P(\infty) ; Z)$ generates the integral ring. In the infinite quarternion projective space, $z(Q P(\infty))$ is generated by 1 and $(\alpha(\sqrt{ } y))^{2}$, where $y \in H^{4}(Q P(\infty) ; Z)$ generates the ring. The $z\left(M^{16}\right)$ for the Caley projective plane is easily computed for any $K$-genus. It is useful to note that for any integer $n, \alpha(n x) \in z(C P(\infty))$ also. If this is applied to the $A$-genus, we have

$$
2[(\sinh 4 x) / 2]+8\left[\left(\sinh ^{2} 2 x\right) / 4\right]+1=\exp (4 x) \in z(C P(\infty)) .
$$

If we regard the integral ring of the classifying space, $B(U(n))$, of a unitary group as generated by the elementary symmetric functions $\left(1+x_{1}\right) \cdots\left(1+x_{n}\right)$, then $z(B(U(n)))$ is correspondingly generated by "elementary symmetric functions" $\left(1+\alpha\left(x_{1}\right)\right)\left(1+\alpha\left(x_{2}\right)\right) \cdots$ $\left(1+\alpha\left(x_{n}\right)\right)$; that is, by $1, \alpha\left(x_{1}\right)+\alpha\left(x_{2}\right)+\cdots+\alpha\left(x_{n}\right), \cdots$, $\alpha\left(x_{1}\right) \cdots \alpha\left(x_{n}\right)$. If we now regard the element $\alpha\left(x_{1}\right)+\cdots+\alpha\left(x_{n}\right)$ $\in z(B(U(n)))$ as an analog of the Chern character, which is naturally associated with the Todd genus, then we obtain extensions for any genus of the integrality relations, based on the Todd genus, which were found in $[2$, Chapter VII] for unitary bundles over closed oriented manifolds. It is not difficult to extend this note to cover the $\hat{A}$-genus. The role of $Z$ is played by the ring of fractions of the form $n / 2^{j}$, but otherwise there is no appreciable difference. We ask if, using a complex bordism theory based on $M U[1]$, it is possible to find a similar analysis of the Todd genus itself.

\section{REFERENCES}

1. M. F. Atiyah, Bordism and cobordism, Proc. Cambridge Philos. Soc. 57 (1961), 200-208. 
2. A. Borel and F. Hirzebruch, Characteristic classes and homogeneous spaces. II, Amer. J. Math. 81 (1959), 315-382.

3. E. H. Brown, Cohomology theories, Ann. of Math. (2) 75 (1962), 467-484.

4. P. E. Conner and E. E. Floyd, Differentiable periodic maps, Bull. Amer. Math. Soc. 68 (1962), 76-86.

5. E. Dyer, Relations between cohomology theories, Colloquium on Algebraic Topology, mimeographed notes, Aarhus Univ. (1962), 89-93.

6. F. Hirzebruch, Neue topologische Methoden in der algebraische Geometrie, Ergebnisse Math., No. 9, Springer, Berlin, 1956.

7. J. W. Milnor, Lectures on characteristic classes, mimeographed notes, Princeton Univ., Princeton, N. J.

8. G. W. Whitehead, Generalized homology theories, Trans. Amer. Math. Soc. 102 (1962), 227-283.

Massachusetts Institute of Technology 